P75 (continued)

Objective: Habitually eating late dinners is considered unhealthy as it may cause other unhealthy behaviors; therefore, we examine the relationship between eating late dinners and health-related behaviors among Japanese workers.

Design, Setting, and Participants: In 2015, we conducted a cross-sectional survey on 1521 Japanese drivers (1432 men, 89 women) working at a courier company.

Outcome Measures and Analysis: We compared the demographic, lifestyle-related (e.g., eating dinner within two hours before sleeping, skipping breakfast, sleeping patterns, smoking, alcohol consumption), and healthcheckup information (e.g., body mass index, blood sugar, blood pressure) of drivers who ate late dinners at least three days per week ( $\geq 3$ days) and those who ate late dinners less than three days per week ( $<3$ days) using chisquare tests.

Results: The $\geq 3$ days group comprised more men, individuals in their forties, and married and overtime workers. there were no differences in health checkup indexes between the two groups. However, more drivers in the $\geq 3$ days group drank alcohol at night everyday (35.4\% vs $23.9 \%$, p $<0.001)$, skipped breakfast (44.2\% vs $39.1 \%$, $\mathrm{p}=0.05)$, ate hurriedly (36.2\% vs $22.1 \%, \mathrm{p}<0.001)$, and did not sleep well $(38.2 \%$ vs $19.9 \%, \mathrm{p}<0.001)$ compared to the $<3$ days group.

Conclusions and Implications: Considering that eating late dinners co-occurred with other health-risk behaviors, interventions to change dinnertime must be implemented in order to encourage workers to eat breakfasts regularly. This may also result in reduced overtime work, implying that successful behavior change will involve considering company policies.

Funding: None

\section{P76 One-on-One Education Increases Breastfeeding Initiation Rates in the Hospital} Martha Archuleta,PhD, RDN, martha.archuleta@usu.edu, Utah State University, 2500 South State Street, Salt Lake City, UT 84105; J. Kudin, MS, RDN; C. Grace, MDA, RDN, CBE; J. Odei, PhD; M. Graf, MS, RDN; M. Smith, MS, RDN

Objective: To evaluate the effectiveness of one-on-one breastfeeding education by a dietitian that is a certified breastfeeding educator on breastfeeding initiation rates in the hospital.

Design, Setting, Participants, and Intervention: A quasi-experimental design with a convenience sample was used. Mothers delivering infants at Siloam Springs Regional Hospital over a six month period were in the control group (written education only; $n=60$ ) or intervention group (written and one-on-one education; $n=60$ ).

Outcome Measures and Analysis: A chart review was conducted to collect demographic and other study-related data. Data were analyzed using logistical regression and resulting 95\% confidence intervals. Addition of one-on-one education, demographics and other variables were assessed for association with breastfeeding initiation. Study methods were approved by the Utah State University IRB.
Results: One-on-one education while in the hospital for delivering the infant, increased the odds of breastfeeding initiation by 20 times. However, planning to breastfeed prior to giving birth increased the odds for breastfeeding initiation the most by far, with these women being over 200 times more likely to initiate breastfeeding. Other factors influencing breastfeeding initiation included: maternal education level, employment status and parity. Conclusion and Implications: One-on-one breastfeeding education by a certified breastfeeding educator can be a cost-effective intervention for improving breastfeeding initiation rates in hospitals, especially if the education is conducted by a dietitian already on staff. Further, because of the strong association of plans to breastfeed, with actually initiating breastfeed, prenatal breastfeeding education is of high importance.

Funding: None

\section{P77 Curriculum Equips Nutrition Majors to Share MyPlate on Campus}

Sandra Baker,EdD, RD, bakers@udel.edu, University of

Delaware, Carpenter Sports Building, Newark, DE 19716

Objective: To describe the development of a course equipping nutrition majors to teach the MyPlate and SuperTracker tools to fellow students and discuss its effects.

Target Audience: Upperclassmen nutrition majors $(n=8)$ were chosen to enroll in an experimental one-credit class. Students created and gave 4 presentations to 181 peers.

Theory, Prior Research, Rationale: Social Cognitive Theory maintains that individuals learn by observing models similar to themselves. The USDA's ChooseMy Plate.gov site has considerable resources regarding healthy eating. Nutrition majors may be especially suited to share the tools with fellow students.

Description of Course and Curriculum: The first weeks of the 15 week course included review of MyPlate and SuperTracker. Next, best practices for developing engaging presentations was discussed. Students then created 30 minute presentations with topics relevant to the college population. They were required to include: a description of MyPlate, review of ChooseMyPlate.gov website resources and demonstration of SuperTracker. After practice and peer critique, students presented in classes with non-nutrition majors. Students developed a pre and post survey evaluating the effectiveness of the presentations.

Evaluation: Using a 5-point scale, course evaluations were favorable with mean $=4.31 / 5.0$. One representative student comment "This was unlike any course I have taken. I grew in my presentation skills and benefited from the professors suggestions." The results of the pre and post survey were also positive with students suggesting that SuperTracker should be demonstrated across campus. Conclusions and Implications: Nutrition majors benefited from the process of planning and conducting the presentations and peers received the presentations favorably. The university's nutrition program plans to

Continued on page S39 